Math. Res. Lett.

Volume 22, Number 5, 1541-1562, 2015

\title{
A new Fourier transform
}

\author{
JONATHAN WANG
}

\begin{abstract}
In order to define a geometric Fourier transform, one usually works with either $\ell$-adic sheaves in characteristic $p>0$ or with $\mathcal{D}$-modules in characteristic 0 . If one considers $\ell$-adic sheaves on the stack quotient of a vector bundle $V$ by the homothety action of $\mathbb{G}_{m}$, however, Laumon provides a uniform geometric construction of the Fourier transform in any characteristic. The category of sheaves on $\left[V / \mathbb{G}_{m}\right]$ is closely related to the category of (unipotently) monodromic sheaves on $V$. In this article, we introduce a new functor, which is defined on all sheaves on $V$ in any characteristic, and we show that it restricts to an equivalence on monodromic sheaves. We also discuss the relation between this new functor and Laumon's homogeneous transform, the Fourier-Deligne transform, and the usual Fourier transform on $\mathcal{D}$-modules (when the latter are defined).
\end{abstract}

\section{Introduction}

In order to define a geometric Fourier transform, one usually works with either $\ell$-adic sheaves in characteristic $p>0$ or with $\mathcal{D}$-modules in characteristic 0 (under these conditions one has a rank 1 local system on $\mathbb{A}^{1}$ which plays the role of the function $e^{i x}$ in classical Fourier analysis). If one only needs to consider homogeneous sheaves, however, Laumon [10] provides a uniform geometric construction of the Fourier transform for $\ell$-adic sheaves in any characteristic. Laumon considers homogeneous sheaves as sheaves on the stack quotient of a vector bundle $V$ by the homothety $\mathbb{G}_{m}$ action. This category is closely related to the category of (unipotently) monodromic sheaves on $V$ (cf. [5]). While it has been well known to experts that a similar uniform construction of the Fourier transform exists for monodromic sheaves (Beilinson suggests a definition in [4, footnote 2]), the details have not been exposited in the literature. In this note, we fill in this gap. We also introduce a new functor, which is defined on all sheaves in any characteristic, and show that it agrees with the usual Fourier transform on monodromic sheaves. 
We define the new Fourier transform Four $_{B}$ in $\S 2$ and show that the "square" Four $_{B}^{2}$ has a simple formula. In $\S 3$, we use this formula to prove the main result that $\operatorname{Four}_{B}$ induces an equivalence of bounded derived categories of monodromic (étale) sheaves. We also discuss the relation between Four $_{B}$ and Laumon's homogeneous Fourier transform. In $\S 4$, we compare Four $_{B}$ and the Fourier-Deligne transform in characteristic $p>0$. Our study of Four $_{B}$ reveals several surprising facts about a certain object $j^{*} B$ of the monoidal category $D_{c t f}\left(\mathbb{G}_{m}\right)$. In $\S 5$, we prove the analogous facts about $j^{*} B$ in the $\mathcal{D}$-module setting by considering the Mellin transform. We use this to show that $\operatorname{Four}_{B}$ agrees with the Fourier transform on monodromic $\mathcal{D}$-modules.

\subsection{Notation and terminology}

Let $k$ be an arbitrary base field and fix an algebraic closure $\bar{k}$. Choose a prime $\ell$ not equal to the characteristic of $k$. Let $R$ be a finite commutative $\mathbb{Z} / \ell^{r}$-algebra for a positive integer $r$. Fix a base scheme $S$ of finite type over $k$. Let $\pi: V \rightarrow S$ be a vector bundle of rank $d$ and $\pi^{\vee}: V^{\vee} \rightarrow S$ the dual vector bundle. We will work with the bounded derived category $D_{c}^{b}(V)=D_{c}^{b}(V, R)$ of étale sheaves of $R$-modules with constructible cohomologies. Our results are also true when $D_{c}^{b}(V, R)$ is replaced by the full subcategory $D_{c t f}(V, R)$ consisting of complexes with finite Tor-dimension, or by $D_{c}^{b}\left(V, \overline{\mathbb{Q}}_{\ell}\right)$. All functors will be assumed to be derived.

We say a complex $M \in D_{c}^{b}(V)$ is monodromic if $M$ is monodromic in the sense of Verdier [13] after base change to $\bar{k}$. This is equivalent to the existence of an integer $n$ coprime to $p$ and an isomorphism $\theta(n)^{*} M \cong \operatorname{pr}_{2}^{*} M$ where $\theta(n): \mathbb{G}_{m} \times V \rightarrow V$ sends $(\lambda, v)$ to $\lambda^{n} v$, and $\mathrm{pr}_{2}: \mathbb{G}_{m} \times V \rightarrow V$ is the projection [13, Proposition 5.1]. We denote the monodromic subcategory by $D_{\text {mon }}^{b}(V)$. We recall the fact that $\pi_{!} \cong 0$ ! on monodromic complexes (cf. [13, Lemme 6.1] or [12, Proposition 1] for two different methods of proof), where $0: S \hookrightarrow V$ is the zero section.

The category $D_{c t f}\left(\mathbb{G}_{m}\right)$ of étale sheaves is monoidal with respect to convolution with compact support, which is defined by

$$
L * K=m_{!}(L \otimes K)
$$

where $m: \mathbb{G}_{m} \times \mathbb{G}_{m} \rightarrow \mathbb{G}_{m}$ is multiplication, and $L, K \in D_{c t f}\left(\mathbb{G}_{m}\right)$. This monoidal category acts on $D_{c}^{b}(V)$ by

$$
L * M=\theta(1) !(L \otimes M)
$$

where $\theta(1): \mathbb{G}_{m} \times V \rightarrow V$ is the action map, $L \in D_{c t f}\left(\mathbb{G}_{m}\right)$, and $M \in D_{c}^{b}(V)$. 


\section{The functor $\operatorname{Four}_{B}$ and its square}

Let $u: \mathbb{A}^{1}-\{1\} \hookrightarrow \mathbb{A}^{1}$ be the open embedding removing $1 \in \mathbb{A}^{1}(k)$, and let $j: \mathbb{A}^{1}-\{0\} \hookrightarrow \mathbb{A}^{1}$ be the open embedding removing zero. Define

$$
B=u_{*} R \in D_{c t f}\left(\mathbb{A}^{1}\right)
$$

One observes that $h_{!} B=0$ where $h: \mathbb{A}^{1} \rightarrow$ Spec $k$ is the structure map, and $0^{*} B \cong R$ where $0: \operatorname{Spec} k \hookrightarrow \mathbb{A}^{1}$.

Define $\operatorname{Four}_{V / S, B}: D_{c}^{b}(V) \rightarrow D_{c}^{b}\left(V^{\vee}\right)$ by

$$
\operatorname{Four}_{V / S, B}(M)=\operatorname{pr}_{!}^{\vee}\left(\operatorname{pr}^{*} M \otimes \mu^{*} B\right)[d]
$$

where $\operatorname{pr}^{\vee}: V^{\vee} \times_{S} V \rightarrow V^{\vee}$ and pr: $V^{\vee} \times_{S} V \rightarrow V$ are the projections and $\mu: V^{\vee} \times_{S} V \rightarrow \mathbb{A}^{1}$ is the natural pairing $(\xi, v) \mapsto\langle v, \xi\rangle$. This is the new Fourier transform that we will consider. Our goal in this section is to prove the following theorem.

Theorem 2.1. There is a canonical isomorphism

$$
\operatorname{Four}_{V^{\vee} / S, B} \circ \operatorname{Four}_{V / S, B}(M) \cong j^{*} B * M(-d)[1] .
$$

for $M \in D_{c}^{b}(V)$.

Let $\mathrm{pr}^{\prime}, \mathrm{pr}^{\prime \prime}: V \times_{S} V \rightarrow V$ be the first and second projections, respectively, and $\operatorname{pr}_{i j}$ the projection from $V \times{ }_{S} V^{\vee} \times{ }_{S} V$ to the product of the $i$ 'th and $j$ 'th factor. The usual formal argument shows that $\operatorname{Four}_{V^{\vee} / S, B}{ }^{\circ}$ $\operatorname{Four}_{V / S, B}$ is isomorphic to the functor $M \mapsto \mathrm{pr}_{!}^{\prime}\left(\mathrm{pr}^{\prime \prime *} M \otimes K\right)$ where

$$
K=\operatorname{pr}_{13 !}\left(\operatorname{pr}_{12}^{*} \mu^{*} B \otimes \operatorname{pr}_{23}^{*} \mu^{*} B\right)[2 d] .
$$

We claim there exists a canonical isomorphism

$$
K \cong \rho ! \operatorname{pr}_{1}^{*} j^{*} B(-d)[1]
$$

where $\rho: \mathbb{G}_{m} \times V \rightarrow V \times_{S} V$ is defined by $(\lambda, v) \mapsto(\lambda v, v)$, and $\operatorname{pr}_{1}: \mathbb{G}_{m} \times$ $V \rightarrow \mathbb{G}_{m}$ is the natural projection. This claim implies the theorem since $\operatorname{pr}_{!}^{\prime}\left(\operatorname{pr}^{\prime \prime *} M \otimes \rho ! \operatorname{pr}_{1}^{*} j^{*} B\right) \cong j^{*} B * M$ by the projection formula.

We first establish two lemmas which will help us prove the claim.

Lemma 2.2. If $v, w \in V(\bar{k})$ are not in the same $\mathbb{G}_{m}$-orbit, then the stalk $K_{(v, w)}$ equals 0 . 
Proof. We can assume $S=\operatorname{Spec} \bar{k}$. Clearly $v$ and $w$ cannot both be zero; we will assume $v \neq 0$. Since $v, w$ are not in the same $\mathbb{G}_{m}$-orbit, there exists $\xi \in V^{\vee}(\bar{k})$ such that $\langle w, \xi\rangle=0$ and $\langle v, \xi\rangle \neq 0$. Let $\langle v\rangle: V^{\vee} \rightarrow \mathbb{A}_{\bar{k}}^{1}$ denote the evaluation by $v$ map. Split $V^{\vee}$ as $\bar{k} \xi \oplus H_{v}$ where $H_{v}=(\bar{k} v)^{\perp}$. With respect to this decomposition, $\langle v\rangle^{*} B \otimes\langle w\rangle^{*} B \cong B \otimes\left(\left.\langle w\rangle\right|_{H_{v}}\right)^{*} B$. Then by Künneth formula,

$$
\pi_{!}^{\vee}\left(\langle v\rangle^{*} B \otimes\langle w\rangle^{*} B\right) \cong h_{!} B \otimes\left(\left.\pi^{\vee}\right|_{H_{v}}\right) !\left(\left.\langle w\rangle\right|_{H_{v}}\right)^{*} B=0
$$

Therefore $K_{(v, w)}=0$.

Lemma 2.3. There is a canonical isomorphism

$$
J^{*} K \cong J^{*} \rho ! \operatorname{pr}_{1}^{*} j^{*} B(-d)[1]
$$

where $J: V \times{ }_{S} V-0(S) \hookrightarrow V \times{ }_{S} V$ is the open embedding removing zero.

Proof. We use $V^{\circ}$ to denote $V-0(S)$. In this proof we will use $\rho$ to denote the restricted morphism $\mathbb{G}_{m} \times V^{\circ} \hookrightarrow V \times{ }_{S} V$, which is an immersion, and $\operatorname{pr}_{1}: \mathbb{G}_{m} \times V^{\circ} \rightarrow \mathbb{G}_{m}$ to denote the projection. From Lemma 2.2 we know that $J^{*} K$ is supported on the image of $\rho$. Thus it suffices to consider $\rho^{*} J^{*} K$. Define

$$
\omega: \mathbb{G}_{m} \times V^{\vee} \underset{S}{\times} V^{\circ} \rightarrow \mathbb{G}_{m} \times \mathbb{A}^{1} \times V^{\circ}
$$

by sending $(\lambda, \xi, v)$ to $(\lambda,\langle v, \xi\rangle, v)$. Then

$$
\rho^{*} J^{*} K \cong \operatorname{pr}_{13 !} \omega ! \omega^{*} \operatorname{pr}_{12}^{*}\left(m^{*} B \otimes p_{2}^{*} B\right)[2 d]
$$

where $\mathrm{pr}_{13}, \mathrm{pr}_{12}$ are projections from $\mathbb{G}_{m} \times \mathbb{A}^{1} \times V^{\circ}$ and $m, p_{2}: \mathbb{G}_{m} \times \mathbb{A}^{1} \rightarrow$ $\mathbb{A}^{1}$ are the multiplication and projection maps. Since $\omega$ is in fact a vector bundle of rank $d-1$, we see that $\omega_{!} R$ is isomorphic to $R(1-d)[2-2 d]$. Therefore the projection formula implies that

$$
\rho^{*} J^{*} K \cong \operatorname{pr}_{13 !} \operatorname{pr}_{12}^{*}\left(m^{*} B \otimes p_{2}^{*} B\right)(1-d)[2] .
$$

We have a Cartesian square

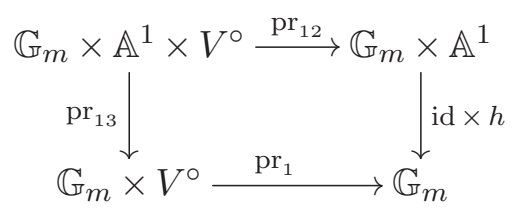


so proper base change gives $\operatorname{pr}_{13 !} \operatorname{pr}_{12}^{*} \cong \operatorname{pr}_{1}^{*}(\mathrm{id} \times h)$ !. We have an exact triangle

$$
R \rightarrow p_{2}^{*} B \rightarrow(\mathrm{id} \times 1) ! R(-1)[-1]
$$

where $1: \operatorname{Spec} k \hookrightarrow \mathbb{A}^{1}$ is the complement of $u$. Since $(\mathrm{id} \times h) !\left(m^{*} B\right)=0$ by a change of variables, we deduce that

$$
\begin{aligned}
(\mathrm{id} \times h)_{!}\left(m^{*} B \otimes p_{2}^{*} B\right) & \cong(\mathrm{id} \times h)_{!}\left(m^{*} B \otimes(\mathrm{id} \times 1) ! R\right)(-1)[-1] \\
& \cong j^{*} B(-1)[-1] .
\end{aligned}
$$

Now it follows that $\rho^{*} J^{*} K \cong \operatorname{pr}_{1}^{*} j^{*} B(-d)[1]$.

Proof of Theorem 2.1. The case $d=0$ is obvious since $h_{!} B=0$ and $0^{*} B \cong$ $R$. From now on we will assume that $d>0$. We will show that both sides of (2.1.1) are in the essential image of the functor $\tau_{\leq 0} J_{*} J^{*}$, i.e., there are isomorphisms

$$
K \cong \tau_{\leq 0} J_{*} J^{*}(K) \text { and } \rho ! \operatorname{pr}_{1}^{*} j^{*} B(-d)[1] \cong \tau_{\leq 0} J_{*} J^{*}\left(\rho ! \operatorname{pr}_{1}^{*} j^{*} B(-d)[1]\right) \text {. }
$$

The claimed existence of an isomorphism (2.1.1) will then follow from Lemma 2.3 .

A stalk computation shows that $\rho ! \mathrm{pr}_{1}^{*} j^{*} B(-d)[1]$ lives in non-positive degrees. We claim that the natural morphism

$$
\rho ! \operatorname{pr}_{1}^{*} j^{*} B(-d)[1] \rightarrow \tau_{\leq 0}\left(J_{*} J^{*} \rho ! \operatorname{pr}_{1}^{*} j^{*} B(-d)[1]\right)
$$

is an isomorphism. Let $0: S \hookrightarrow V \times_{S} V$ denote the zero section. From the exact triangle $0 ! 0^{!} \rightarrow \mathrm{id} \rightarrow J_{*} J^{*}$, it suffices to show that $0^{!} \rho_{!} \operatorname{pr}_{1}^{*} j^{*} B \in D_{c}^{>2}(S)$. Observe that $\rho$ is $\mathbb{G}_{m}$-equivariant with respect to the $\mathbb{G}_{m}$-action on the second coordinate of $\mathbb{G}_{m} \times V$ and the diagonal action of $\mathbb{G}_{m}$ on $V \times_{S} V$. This implies that $\rho ! \operatorname{pr}_{1}^{*} j^{*} B$ is monodromic. Thus

$$
0^{!} \rho ! \operatorname{pr}_{1}^{*} j^{*} B \cong h_{!} j_{!} j^{*} B(-d)[-2 d] \cong R(-d)[-2 d-1] .
$$

Therefore $0 ! \rho ! \operatorname{pr}_{1}^{*} j^{*} B \in D_{c}^{>2 d}(S)$.

One easily sees that $K_{(0,0)} \cong R(-d)$. Thus $K$ lives in non-positive cohomological degrees. To show that the natural morphism $K \rightarrow \tau_{\leq 0} J_{*} J^{*} K$ is an isomorphism, it suffices by the same argument as above to prove $0 ! K \in D_{\bar{c}}^{\geq 2 d}(S)$. One observes from the definition of $K$ that $K$ is monodromic 
with respect to the diagonal $\mathbb{G}_{m}$-action on $V \times_{S} V$. Therefore

$$
0 ! K \cong \widetilde{\pi}_{!}\left(\operatorname{pr}_{12}^{*} \mu^{*} B \otimes \operatorname{pr}_{23}^{*} \mu^{*} B\right)[2 d]
$$

where $\tilde{\pi}: V \times{ }_{S} V^{\vee} \times{ }_{S} V \rightarrow S$ is the structure map. By projection formula and proper base change, the right hand side is isomorphic to

$$
\pi_{!}^{\prime}\left(\mu^{*} B \otimes \operatorname{pr}^{\vee, *} \operatorname{pr}_{!}^{\vee} \mu^{*} B\right)[2 d]
$$

for $\pi^{\prime}: V \times_{S} V^{\vee} \rightarrow S$ the structure map. The fact that $h_{!} B=0$ implies that $\operatorname{pr}_{!}^{\vee} \mu^{*} B$ is supported at $0(S) \subset V^{\vee}$, and $0^{*} \operatorname{pr}_{!}^{\vee} \mu^{*} B \cong R(-d)[-2 d]$. We deduce that

$$
0 ! K \cong \pi ! R(-d) \cong R(-2 d)[-2 d],
$$

which proves the claim, and hence the theorem.

\section{Properties of Four $_{B}$}

Remark 3.1. The functor $\operatorname{Four}_{V / S, B}$ is not an equivalence on $D_{c}^{b}(V) \rightarrow$ $D_{c}^{b}\left(V^{\vee}\right)$. Consider the one-dimensional case $V=\mathbb{A}_{S}^{1}$. Then $\operatorname{Four}_{V / S, B}(0 ! R)=$ $R[1]$ and $\operatorname{Four}_{V / S, B}\left(1_{!} R\right)=B[1]$. We have $\operatorname{Hom}(R, B) \neq 0$ but $\operatorname{Hom}(0 ! R$, $\left.1_{!} R\right)=0$, so Four $_{V / S, B}$ is not fully faithful.

\subsection{Relation to quotient stacks}

Let $p: V \rightarrow \mathcal{V}=\left[V / \mathbb{G}_{m}\right]$ and $p^{\vee}: V^{\vee} \rightarrow \mathcal{V}^{\vee}=\left[V^{\vee} / \mathbb{G}_{m}\right]$ denote the canonical projections to the quotient stacks. By [10, Lemme 3.2], Laumon's homogeneous transform $\operatorname{Four}_{\mathcal{V} / S}: D_{c}^{b}(\mathcal{V}) \rightarrow D_{c}^{b}\left(\mathcal{V}^{\vee}\right)$ is canonically isomorphic to the functor

$$
K \mapsto \operatorname{pr}_{!}^{\vee}\left(\operatorname{pr}^{*} K \otimes \mu^{*} f_{!} B_{S}\right)[d]
$$

where $f: \mathbb{A}_{S}^{1} \rightarrow \mathcal{A}_{S}$ is the quotient morphism and $B_{S}$ denotes the base change of $B$ from $\mathbb{A}_{k}^{1}$ to $\mathbb{A}_{S}^{1}$. We abuse notation and use $\mathrm{pr}^{\vee}: \mathcal{V}^{\vee} \times_{S} \mathcal{V} \rightarrow$ $\mathcal{V}^{\vee}$, pr: $\mathcal{V}^{\vee} \times_{S} \mathcal{V} \rightarrow \mathcal{V}$, and $\mu: \mathcal{V}^{\vee} \times_{S} \mathcal{V} \rightarrow \mathcal{A}_{S}$ to also denote the induced maps on stacks.

Proposition 3.3. The composed functors

$$
\left(p^{\vee}\right)^{*} \circ \operatorname{Four}_{\mathcal{V} / S} \text { and } \operatorname{Four}_{V / S, B} \circ p^{*}: D_{c}^{b}(\mathcal{V}) \rightarrow D_{c}^{b}\left(V^{\vee}\right)
$$

are canonically isomorphic. 
Proof. The proposition follows from (3.2.1) by applying proper base change to the Cartesian squares
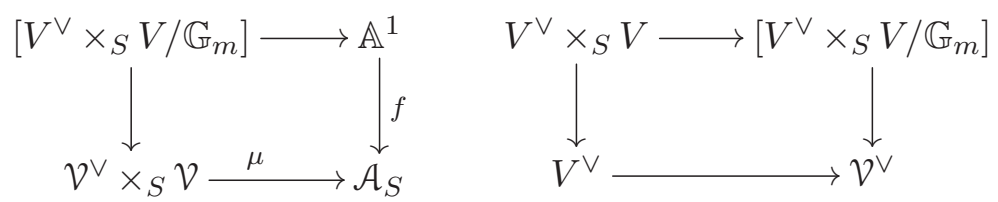

where $\mathbb{G}_{m}$ acts on $V^{\vee} \times_{S} V$ anti-diagonally.

Proposition 3.4. Let $V^{\prime}=V \times \mathbb{A}^{1}$ and let $\mathbb{G}_{m}$ act on both $V$ and $\mathbb{A}^{1}$. We have a canonical open embedding $\nu: V \hookrightarrow\left[V^{\prime} / \mathbb{G}_{m}\right]: v \mapsto(v, 1)$. Similarly, we have $\nu^{\vee}: V^{\vee} \hookrightarrow\left[\left(V^{\prime}\right)^{\vee} / \mathbb{G}_{m}\right]$ defined by $\nu^{\vee}(\xi)=(\xi,-1)$. The composed functor

$$
D_{c}^{b}(V) \stackrel{\nu_{1}}{\longrightarrow} D_{c}^{b}\left(\left[V^{\prime} / \mathbb{G}_{m}\right]\right) \stackrel{\operatorname{Four}_{\left[V^{\prime} / \mathbb{G}_{m}\right] / S}^{\longrightarrow}}{\longrightarrow} D_{c}^{b}\left(\left[\left(V^{\prime}\right)^{\vee} / \mathbb{G}_{m}\right]\right) \stackrel{\left(\nu^{\vee}\right)^{*}}{\longrightarrow} D_{c}^{b}\left(V^{\vee}\right)
$$

is isomorphic to Four $_{V / S, B}$.

Proof. Observe that $\nu$ factors into the composition of an open affine chart $V \hookrightarrow \mathbb{P}\left(V^{\prime}\right)$ and the open embedding $\mathbb{P}\left(V^{\prime}\right)=\left[\left(V^{\prime}-0(S)\right) / \mathbb{G}_{m}\right] \hookrightarrow\left[V^{\prime} / \mathbb{G}_{m}\right]$. Similarly, we have a factorization of $\nu^{\vee}$. The proposition now follows from [10, Proposition 1.6], since the restriction of the incidence hyperplane in $\mathbb{P}\left(\left(V^{\prime}\right)^{\vee}\right) \times_{S} \mathbb{P}\left(V^{\prime}\right)$ to $V^{\vee} \times_{S} V$ is $\mu^{-1}(\{1\})$.

\subsection{An equivalence induced by $\operatorname{Four}_{V / S, B}$}

Let $p: V \rightarrow \mathcal{V}$ be as in the previous subsection.

Proposition 3.6. Let $\mathcal{C}_{V}$ denote the full subcategory of $D_{c}^{b}(V)$ consisting of complexes $M$ such that $p_{!} M=0$. The functor $\operatorname{Four}_{V / S, B}$ induces an equivalence $\mathcal{C}_{V} \rightarrow \mathcal{C}_{V^{\vee}}$.

Proof. Proper base change and projection formula imply that Four $_{V / S, B}$ sends $\mathcal{C}_{V}$ to $\mathcal{C}_{V}$ and vice versa. We also see by proper base change that $p^{*} p_{!} M \cong R * M$ for $M \in D_{c}^{b}(V)$, where $R$ is the constant sheaf on $\mathbb{G}_{m}$. From the exact triangle $1 ! R(-1)[-2] \rightarrow R \rightarrow B$ we deduce that $j^{*} B * M \cong$ $M(-1)[-1]$ for $M \in \mathcal{C}_{V}$. Therefore Theorem 2.1 implies that

$$
\operatorname{Four}_{V \vee / S, B} \circ \operatorname{Four}_{V / S, B}(M) \cong M(-d-1)
$$

for $M \in \mathcal{C}_{V}$, and we deduce the proposition. 


\subsection{Monodromic complexes}

We will show that $\operatorname{Four}_{V / S, B}$ also induces an equivalence on the subcategories of monodromic complexes. We use the notation and results of Appendix A.

Theorem 3.8. (i) The functor Four $_{V / S, B}$ preserves monodromicity, and the restriction defines an equivalence $D_{\text {mon }}^{b}(V) \rightarrow D_{\text {mon }}^{b}\left(V^{\vee}\right)$.

(ii) For $N \in D_{\mathrm{mon}}^{b}\left(V^{\vee}\right)$, the pro-object

$$
\operatorname{pr}_{!}\left(\operatorname{pr}^{\vee, *} N \otimes \mu^{*} j_{*} I^{0}\right)(d+1)[d+1]
$$

is essentially constant ${ }^{1}$.

(iii) The functor from $D_{\text {mon }}^{b}\left(V^{\vee}\right)$ to $D_{\text {mon }}^{b}(V)$ defined by (3.8.1) is quasiinverse to $\operatorname{Four}_{V / S, B}$.

Since $B$ is not monodromic, our first step is to compute the "monodromization" of $B$.

Lemma 3.9. There is an isomorphism of pro-objects

$$
I^{0} * B \cong j_{*} I^{1}(-1)[-1] .
$$

Proof. First we show that the restriction $I^{0} * j^{*} B$ is isomorphic to $I^{1}(-1)[-1]$. The exact triangle $1 ! R(-1)[-2] \rightarrow R \rightarrow B$ induces by convolution exact triangles

$$
I_{n}^{0}(-1)[-2] \rightarrow I_{n}^{0} * R \rightarrow I_{n}^{0} * j^{*} B
$$

for $p \nmid n$. Taking "lim" and using Lemma A.4, the first arrow is isomorphic to the augmentation map $I^{0}(-1)[-2] \rightarrow R(-1)[-2]$. Therefore we deduce that the pro-object $I^{0} * j^{*} B$ is isomorphic to $I^{1}(-1)[-1]$.

To complete the proof, it suffices to show that the canonical morphism

$$
I^{0} * B \rightarrow j_{*} j^{*}\left(I^{0} * B\right)
$$

is an isomorphism. This is equivalent to proving that $0^{!}\left(I^{0} * B\right)=0$. Since $I^{0} * B$ is monodromic, $0^{!}\left(I^{0} * B\right) \cong h_{!}\left(I^{0} * B\right)$. By the Kunneth formula, $h_{!}\left(I^{0} * B\right) \cong h_{!} j_{!} I^{0} \otimes h_{!} B=0$.

\footnotetext{
${ }^{1} \mathrm{~A}$ pro-object is essentially constant if it is isomorphic to an object of $D_{\text {mon }}^{b}(V)$, which is considered as a pro-object via the constant embedding.
} 
Proof of Theorem 3.8. One easily sees that $\operatorname{Four}_{V / S, B}$ preserves monodromicity. Theorem 2.1 and Lemma A.4 together imply that for $M \in D_{\text {mon }}^{b}(V)$, we have

$$
\operatorname{Four}_{V^{\vee} / S, B} \circ \operatorname{Four}_{V / S, B}(M) \cong I^{1} * M(-d)[2] .
$$

Since $I^{-1} * I^{1} \cong I^{0}(-1)[-2]$ by Corollary A.9, we deduce that $\operatorname{Four}_{V / S, B}$ is an equivalence, with inverse functor $I^{-1} * \operatorname{Four}_{V^{\vee} / S, B}(d+2)[2]$. Lemmas 3.9 and A.4 imply that for $N \in D_{\text {mon }}^{b}\left(V^{\vee}\right)$, we have isomorphisms

$$
I^{-1} * \operatorname{Four}_{V^{\vee} / S, B}(N) \cong I^{-1} * \operatorname{pr}_{!}\left(\operatorname{pr}^{\vee}, * N \otimes \mu^{*} j_{*} I^{1}\right)[d+1]
$$

Applying Corollary A.9 again, we get (iii).

Remark 3.10. Observe that the formula (3.8.1) is very similar to Beilinson's suggested definition of the monodromic Fourier transform in [4].

Proposition 3.11. The object $j^{*} B \in D_{c t f}\left(\mathbb{G}_{m}\right)$ satisfies the following properties:

1) $j^{*} B$ is not invertible in the monoidal category $D_{c t f}\left(\mathbb{G}_{m}\right)$.

2) $j^{*} B$ is invertible in the quotient of $D_{c t f}\left(\mathbb{G}_{m}\right)$ by the ideal generated by the constant sheaf $R$.

3) There are canonical isomorphisms $I_{n}^{0} * j^{*} B \cong I_{n}^{1}(-1)[-2]$ for $p \nmid n$.

Proof. We showed in Remark 3.1 that $\operatorname{Four}_{\mathbb{A}^{1}, B}$ is not an equivalence on $D_{c}^{b}\left(\mathbb{A}^{1}\right)$. Since $\operatorname{Four}_{\mathbb{A}^{1}, B}^{2}(M)$ is isomorphic to $j^{*} B * M(-1)[1]$, we deduce that $j^{*} B$ is not invertible in the monoidal category $D_{c t f}\left(\mathbb{G}_{m}\right)$.

From the exact triangle $1_{!} R(-1)[-2] \rightarrow R \rightarrow j^{*} B$ on $\mathbb{G}_{m}$, we see that in the quotient of $D_{c t f}\left(\mathbb{G}_{m}\right)$ by the ideal generated by $R$, the object $j^{*} B$ is isomorphic to $1_{!} R(-1)[-1]$, which is invertible.

Lemma 3.9 gives an isomorphism $I^{0} * j^{*} B \cong I^{1}(-1)[-2]$. Convolving with $I_{n}^{0}$, we get an isomorphism $I_{n}^{0} * j^{*} B \cong I_{n}^{0} * I^{1}$. One observes that $I_{n}^{0} *$ $I^{1} \cong I_{n}^{1}(-1)[-2]$ by Corollary A.9.

\section{Relation to Fourier-Deligne transform}

Suppose that $k$ has characteristic $p>0$. Assume that $R$ contains a primitive $p$-th root of unity $\zeta$ (where "primitive" means that $\zeta-1$ is invertible). Let $\psi: \mathbb{F}_{p} \rightarrow R^{\times}$be the corresponding additive character with $\psi(1)=\zeta$, and 
let $\mathcal{L}_{\psi}$ denote the Artin-Schreier sheaf. The usual Fourier-Deligne transform $\operatorname{Four}_{V / S, \mathcal{L}_{\psi}}: D_{c}^{b}(V) \rightarrow D_{c}^{b}\left(V^{\vee}\right)$ is defined by

$$
\operatorname{Four}_{V / S, \mathcal{L}_{\psi}}(M)=\operatorname{pr}_{!}^{\vee}\left(\operatorname{pr}^{*} M \otimes \mu^{*} \mathcal{L}_{\psi}\right)[d] .
$$

Lemma 4.1. There is a canonical isomorphism

$$
\iota^{*} j^{*} \mathcal{L}_{\psi} * \mathcal{L}_{\psi} \cong B[-1]
$$

where $\iota: \mathbb{G}_{m} \rightarrow \mathbb{G}_{m}$ sends $\lambda \mapsto-\lambda^{-1}$.

Proof. By a change of variables, $\iota^{*} j^{*} \mathcal{L}_{\psi} * \mathcal{L}_{\psi}$ is isomorphic to

$$
\operatorname{Four}_{\mathbb{A}^{1}, \mathcal{L}_{\psi^{-1}}}\left(j ! j^{*} \mathcal{L}_{\psi}\right)[-1]
$$

We have an exact triangle

$$
j_{!} j^{*} \mathcal{L}_{\psi} \rightarrow \mathcal{L}_{\psi} \cong \operatorname{Four}_{\mathbb{A}^{1}, \mathcal{L}_{\psi}}\left(1_{!} R[-1]\right) \rightarrow 0_{*} R .
$$

Applying $\operatorname{Four}_{\mathbb{A}^{1}, \mathcal{L}_{\psi^{-1}}}$ and using the Fourier-Deligne inversion formula on the middle term, we have an exact triangle

$$
\operatorname{Four}_{\mathbb{A}^{1}, \mathcal{L}_{\psi-1}}\left(j ! j^{*} \mathcal{L}_{\psi}\right) \rightarrow 1_{!} R(-1)[-1] \rightarrow R[1] .
$$

This induces an isomorphism $\operatorname{Four}_{\mathbb{A}^{1}, \mathcal{L}_{\psi^{-1}}}\left(j ! j^{*} \mathcal{L}_{\psi}\right) \rightarrow u_{*} R=B$. This isomorphism is unique since $\operatorname{Hom}\left(1_{!} R(-1)[-1], R\right)=0$.

Corollary 4.2. In characteristic $p>0$, we have a canonical isomorphism

$$
\operatorname{Four}_{V / S, B}(M) \cong \iota^{*} j^{*} \mathcal{L}_{\psi} * \operatorname{Four}_{V / S, \mathcal{L}_{\psi}}(M)[1] .
$$

\subsection{Monodromization of $\mathcal{L}_{\psi}$ over $\bar{k}$}

We use the notation and results of Appendix A. Suppose that $k$ is algebraically closed, so $A^{0}$ is simply a ring instead of a sheaf of rings (i.e., there is no Galois action).

Lemma 4.4. There exists a (non-canonical) isomorphism of pro-objects

$$
I^{0} * \mathcal{L}_{\psi} \cong j_{*} I^{0}[-1] .
$$


Proof. As in the proof of Lemma 3.9, it suffices to prove the isomorphism after restriction to $\mathbb{G}_{m}$. Let $n$ be coprime to $p$. By proper base change,

$$
1^{*}\left(I_{n}^{0} * j^{*} \mathcal{L}_{\psi}\right) \cong \Gamma_{c}\left(\mathbb{G}_{m}, I_{n}^{0} \underset{R}{\otimes} j^{*} \mathcal{L}_{\psi}\right)
$$

where we observe that the pullback of $I_{n}^{0}$ under the multiplicative inverse map $\mathbb{G}_{m} \rightarrow \mathbb{G}_{m}$ is isomorphic to $I_{n}^{0}$. Since $I_{n}^{0}$ is tamely ramified at $\infty \in \mathbb{P}^{1}(k)$, the canonical map

$$
\Gamma_{c}\left(\mathbb{A}^{1}, j_{!} I_{n}^{0} \otimes \mathcal{L}_{\psi}\right) \rightarrow \Gamma\left(\mathbb{A}^{1}, j_{!} I_{n}^{0} \otimes \mathcal{L}_{\psi}\right)
$$

is an isomorphism (cf. proof of [9, Lemma 7.1(1)]). In particular $\Gamma_{c}\left(\mathbb{G}_{m}, I_{n}^{0} \otimes\right.$ $j^{*} \mathcal{L}_{\psi}$ ) lives in cohomological degrees 0 and 1 . Since $I_{n}^{0} \otimes j^{*} \mathcal{L}_{\psi}$ is locally constant and $\mathbb{G}_{m}$ is not complete, $H_{c}^{0}\left(\mathbb{G}_{m}, I_{n}^{0} \otimes j^{*} \mathcal{L}_{\psi}\right)=0$. Thus $\Gamma_{c}\left(\mathbb{G}_{m}, I_{n}^{0} \otimes\right.$ $\left.j^{*} \mathcal{L}_{\psi}\right)$ lives only in cohomological degree 1 .

We now consider $I_{n}^{0}$ as a locally free sheaf of $A_{n}^{0}$-modules of rank 1 . If we let $\psi^{\prime}$ denote the composition $\mathbb{F}_{p} \rightarrow R^{\times} \rightarrow\left(A_{n}^{0}\right)^{\times}$, then $\mathcal{L}_{\psi} \otimes_{R} A_{n}^{0} \cong$ $\mathcal{L}_{\psi^{\prime}}$, where the latter is the Artin-Schreier sheaf with respect to $\psi^{\prime}$ as a locally free sheaf of $A_{n}^{0}$-modules of rank 1 . Hence $\mathcal{F}:=I_{n}^{0} \otimes_{A_{n}^{0}} j^{*} \mathcal{L}_{\psi^{\prime}}$, which is isomorphic to $I_{n}^{0} \otimes_{R} j^{*} \mathcal{L}_{\psi}$, is a locally free sheaf of $A_{n}^{0}$-modules of rank 1. In particular, $\mathcal{F} \in D_{c t f}\left(\mathbb{G}_{m}, A_{n}^{0}\right)$ and $\Gamma_{c}\left(\mathbb{G}_{m}, \mathcal{F}\right)[1]$ is quasi-isomorphic to a finite projective $A_{n}^{0}$ module $P$. Applying the Grothendieck-Ogg-Shafarevich formula [2, Exposé X, Corollaire 7.2], one checks that the fiber of $P$ over any point of Spec $A_{n}^{0}$ has dimension 1 . So there exists an isomorphism $P \cong A_{n}^{0}$ of $A_{n}^{0}$-modules. Observe from the Cartesian square

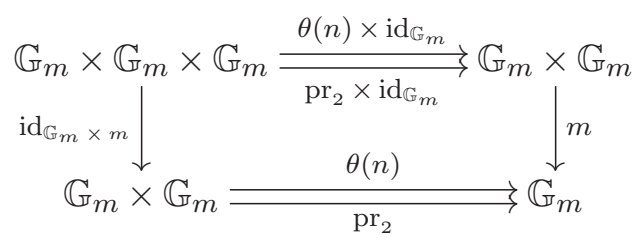

that $I_{n}^{0} * j^{*} \mathcal{L}_{\psi}$ is monodromic, and the monodromy action is induced by the monodromy action on $I_{n}^{0}$. Hence by Corollary A.7, there exists an isomorphism $I_{n}^{0} * j^{*} \mathcal{L}_{\psi}[1] \cong I_{n}^{0}$.

Suppose $n^{\prime}$ is a multiple of $n$ and $p \nmid n^{\prime}$. The kernel $\mathcal{K}$ of the surjection $I_{n^{\prime}}^{0} \rightarrow I_{n}^{0}$ is tamely ramified, so $H_{c}^{2}\left(\mathbb{G}_{m}, \mathcal{K} \otimes j^{*} \mathcal{L}_{\psi}\right)=0$ by the same argument as above. We deduce that

$$
I_{n^{\prime}}^{0} * j^{*} \mathcal{L}_{\psi}[1] \rightarrow I_{n}^{0} * j^{*} \mathcal{L}_{\psi}[1]
$$


is a surjection of sheaves. Since $\left(A_{n^{\prime}}^{0}\right)^{\times} \rightarrow\left(A_{n}^{0}\right)^{\times}$is also surjective, we can find a projective system of isomorphisms $I_{n}^{0} * j^{*} \mathcal{L}_{\psi}[1] \cong I_{n}^{0}$ inducing an isomorphism of pro-sheaves.

Corollary 4.5. When $k$ is algebraically closed, there exists a (non-canonical) isomorphism between the functors $\operatorname{Four}_{V / S, B}$ and $\operatorname{Four}_{V / S, \mathcal{L}_{\psi}}$ restricted to $D_{\text {mon }}^{b}(V) \rightarrow D_{\text {mon }}^{b}\left(V^{\vee}\right)$.

Proof. Lemma 3.9 and Remark A.3 imply that there exists an isomorphism $I^{0} * B \cong j_{*} I^{0}[-1]$. The latter is also isomorphic to $I^{0} * \mathcal{L}_{\psi}$ by Lemma 4.4. One easily sees that the Fourier-Deligne transform preserves monodromicity, and the isomorphism of restricted functors follows from Lemma A.4.

\subsection{The universal Gauss sum}

Let $k$ once again be arbitrary. Define the pro-object

$$
\mathcal{G}=I^{0} * j^{*} \mathcal{L}_{\psi}(1)[1] .
$$

Lemma 4.4 implies that $\mathcal{G}$ is a monodromic pro-sheaf, and there exists a trivialization $\mathcal{G} \cong I^{0}$ after base changing from $k$ to $\bar{k}$. Under the equivalence of abelian categories in Corollary A.7, we see that $\mathcal{G}$ corresponds to an invertible (locally free of rank 1) $A^{0}$-module on Spec $k$. We are motivated by [6, Exposé VI, §4] to think of $\mathcal{G}$ as a "universal Gauss sum".

Define $\iota: \mathbb{G}_{m} \rightarrow \mathbb{G}_{m}$ by $\iota(\lambda)=-\lambda^{-1}$. Then Lemmas 3.9 and 4.1 give a canonical isomorphism

$$
\iota^{*} \mathcal{G} * \mathcal{G} \cong I^{1}[-2] .
$$

We also see that the Fourier-Deligne transform on monodromic complexes is isomorphic to the functor $M \mapsto \operatorname{pr}_{!}^{\vee}\left(\operatorname{pr}^{*} M \otimes \mu^{*} j_{*} \mathcal{G}\right)[d+1]$ on $D_{\text {mon }}^{b}(V) \rightarrow$ $D_{\text {mon }}^{b}\left(V^{\vee}\right)$. By Corollary 4.2, we have

$$
\operatorname{Four}_{V / S, B}(M) \cong \iota^{*} \mathcal{G} * \operatorname{Four}_{V / S, \mathcal{L}_{\psi}}(M)[2] .
$$

for $M$ monodromic.

\section{Relation to Fourier transform on $\mathcal{D}$-modules}

Let $k$ be algebraically closed of characteristic 0 . We use $\mathcal{M}(V)$ to denote the abelian category of quasicoherent right $\mathcal{D}$-modules on $V$. Let $\mathcal{L}=\mathcal{D}_{\mathbb{A}^{1}} /(1-$ 
$\left.\partial_{x}\right) \mathcal{D}_{\mathbb{A}^{1}}$ be the exponential $\mathcal{D}$-module on $\mathbb{A}^{1}=\operatorname{Spec} k[x]$. The Fourier transform is the functor $\operatorname{D\mathcal {M}}(V) \rightarrow \operatorname{D\mathcal {M}}\left(V^{\vee}\right)$ defined by

$$
\operatorname{Four}_{V / S, \mathcal{L}}(M)=\operatorname{pr}_{*}^{\vee}\left(\operatorname{pr}^{!} M \stackrel{!}{\otimes} \mu^{!} \mathcal{L}\right)[1-d]
$$

It is well known [8, Lemme 7.1.4] that this functor can also be described using the isomorphism between the algebras of polynomial differential operators $\mathcal{D}_{V} \vee \rightarrow \mathcal{D}_{V}$ defined in local coordinates by

$$
k\left[\xi_{1}, \ldots, \xi_{d}, \partial_{\xi_{1}}, \ldots, \partial_{\xi_{d}}\right] \rightarrow k\left[v_{1}, \ldots, v_{d}, \partial_{v_{1}}, \ldots, \partial_{v_{d}}\right]: \xi_{i} \mapsto-\partial_{v_{i}}, \partial_{\xi_{i}} \mapsto v_{i}
$$

In the $\mathcal{D}$-module situation, the analog of $B$ is $u_{!} u^{!}\left(\omega_{\mathbb{A}^{1}}\right)$, where $\omega_{\mathbb{A}^{1}}$ is the sheaf of differentials on $\mathbb{A}^{1}$ viewed as a right $\mathcal{D}$-module. We will also call this $\mathcal{D}$-module $B$. A simple calculation shows that ${ }^{2}$

$$
B=k\left[x, \partial_{x}\right] / \partial_{x}(x-1) k\left[x, \partial_{x}\right]
$$

We define $\operatorname{Four}_{V / S, B}: D \mathcal{M}(V) \rightarrow D \mathcal{M}\left(V^{\vee}\right)$ by

$$
\operatorname{Four}_{V / S, B}(M)=\operatorname{pr}_{*}^{\vee}\left(\operatorname{pr}^{!} M \stackrel{!}{\otimes} \mu^{!} B\right)[1-d]
$$

Consider $\operatorname{D\mathcal {N}}\left(\mathbb{G}_{m}\right)$ with the monoidal structure induced by convolution without compact support $L * K:=m_{*}(L \otimes K)$. This monoidal category acts on $D \mathcal{M}(V)$ by $L * M=\theta(1)_{*}(L \otimes M)$. The proof of Lemma 4.1 can be easily modified to prove the following analog of the lemma and Corollary 4.2.

Proposition 5.1. There is a canonical isomorphism

$$
\iota^{*} j^{*} \mathcal{L} * \mathcal{L} \cong B
$$

where $\iota: \mathbb{G}_{m} \rightarrow \mathbb{G}_{m}$ sends $\lambda \mapsto-\lambda^{-1}$. Consequently, we have a canonical isomorphism

$$
\operatorname{Four}_{V / S, B}(M) \cong \iota^{*} j^{*} \mathcal{L} * \operatorname{Four}_{V / S, \mathcal{L}}(M)
$$

\footnotetext{
${ }^{2}$ Beilinson observed that $B$ essentially describes the differential equation for a shift of the Heaviside step function.
} 


\subsection{Mellin transform of $j^{*} B$}

Let $\mathfrak{B}$ denote the Mellin transform of $j^{*} B$, viewed as a $\mathbb{Z}$-equivariant quasicoherent $\mathcal{O}$-module on $\mathbb{A}^{1}=\operatorname{Spec} k[s]$. The Mellin transform functor

$$
\mathfrak{M}: \mathcal{M}\left(\mathbb{G}_{m}\right) \rightarrow \mathrm{QCoh}\left(\mathbb{A}^{1}\right)^{\mathbb{Z}}
$$

is defined by considering $\mathcal{D}\left(\mathbb{G}_{m}\right)$ as the algebra of difference operators

$$
\mathcal{D}=k[s]\left\langle T, T^{-1}\right\rangle /(s T-T(s+1))
$$

under the identifications $s=x \partial_{x}$ and $T=x$. We consider the derived cate-

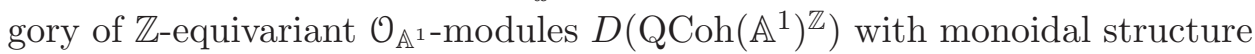
induced by the usual derived tensor product over $k[s]$. This monoidal structure corresponds to the convolution product on $D \mathcal{M}\left(\mathbb{G}_{m}\right)$. More precisely, $\mathfrak{M}(L * K) \cong \mathfrak{M}(L) \otimes_{k[s]} \mathfrak{M}(K)$.

We start by proving the following proposition, which is an analog of Proposition 3.11 in the $\mathcal{D}$-module setting.

Proposition 5.3. The module $\mathfrak{B}$ satisfies the following properties:

1) $\mathfrak{B}$ is not invertible in $D\left(\mathrm{QCoh}\left(\mathbb{A}^{1}\right)^{\mathbb{Z}}\right)$.

2) The restriction of $\mathfrak{B}$ to $\mathbb{A}^{1}-\mathbb{Z}:=\operatorname{Spec} k[s]\left[s^{-1},(s \pm 1)^{-1}, \ldots\right]$ is invertible.

3) For any $\chi \in k$ and $n \in \mathbb{N}$, there exists an isomorphism

$$
\bigoplus_{i \in \mathbb{Z}} k[s] /(s-\chi-i)^{n} \cong \bigoplus_{i \in \mathbb{Z}} \mathfrak{B} \underset{k[s]}{\otimes} k[s] /(s-\chi-i)^{n}
$$

of $\mathcal{D}$-modules, where $T$ acts on $k[s]$ by translation.

In order to prove the proposition, we will need an explicit description of $\mathfrak{B}$. Consider $k(s)$ as a right $\mathcal{D}$-module where $T$ acts by translation. Let $\mathfrak{B}^{\prime}$ denote the $\mathcal{D}$-submodule of $k(s)$ generated by $\frac{1}{s}$, or equivalently, the $k[s]$-submodule generated by $\frac{1}{s+i}$ for all $i \in \mathbb{Z}$.

Lemma 5.4. There exists an isomorphism of $\mathcal{D}$-modules $\mathfrak{B} \cong \mathfrak{B}^{\prime}$. 
Proof. We have $\partial_{x} x=x \partial_{x}+1$ so $\partial_{x}(x-1)=(s+1)-T^{-1} s$ in $\mathcal{D}$. Therefore

$$
\mathfrak{B}=\mathcal{D} /\left((s+1)-T^{-1} s\right) \mathcal{D} .
$$

Let 1 denote the generator of $\mathfrak{B}$. Conjugating $s T=T(s+1)$ in $\mathcal{D}$ by $T^{-1}$ gives $T^{-1} s=(s+1) T^{-1}$ in $\mathcal{D}$. Using this equality, $\mathbf{1}(s+1)=\mathbf{1} T^{-1} s=\mathbf{1}(s+$ 1) $T^{-1}$ in $\mathfrak{B}$, and acting on the right by $T$ gives $\mathbf{1}(s+1) T=\mathbf{1}(s+1)$. Using these relations, we deduce that $\mathfrak{B}$ is generated over $k$ by $1 T^{i}$ for $i \in \mathbb{Z}$ and $\mathbf{1} s^{j}$ for $j>0$. Then $\mathbf{1} \mapsto \frac{1}{s+1}$ defines a morphism of $\mathcal{D}$-modules $\mathfrak{B} \rightarrow k(s)$. Since $\frac{1}{s+i}$ for $i \in \mathbb{Z}$ and $s^{j}$ for $j \geq 0$ are $k$-linearly independent in $k(s)$, we see that this morphism is an injection $\mathfrak{B} \hookrightarrow k(s)$. The image is $\mathfrak{B}^{\prime}$.

Proof of Proposition 5.3. Suppose that $\mathfrak{B}$ is invertible in $D\left(\mathrm{QCoh}\left(\mathbb{A}^{1}\right)^{\mathbb{Z}}\right)$, i.e., there exists an object $N$ of this monoidal category such that $\mathfrak{B} \otimes_{k[s]} N \cong$ $k[s]$. Then $N \cong \operatorname{Hom}_{k[s]}(k[s], N) \cong \operatorname{Hom}_{k[s]}(\mathfrak{B}, k[s])$. There are no nonzero morphisms from $\mathfrak{B}^{\prime}$ to $k[s]$, so $H^{0} N=0$. On the other hand, since $k(s) \otimes_{k[s]}$ $\mathfrak{B}^{\prime} \cong k(s)$, we have $k(s) \otimes_{k[s]} N \cong k(s)$, which implies that $H^{0} N \neq 0$. We thus get a contradiction, so $\mathfrak{B}$ is not invertible.

Since $\mathcal{O}\left(\mathbb{A}^{1}-\mathbb{Z}\right)=k[s]\left[s^{-1},(s \pm 1)^{-1}, \ldots\right] \subset k(s)$, we see that

$$
\mathcal{O}\left(\mathbb{A}^{1}-\mathbb{Z}\right) \underset{k[s]}{\otimes} \mathfrak{B}^{\prime}=\mathcal{O}\left(\mathbb{A}^{1}-\mathbb{Z}\right) \subset k(s)
$$

is the identity object, proving (2).

The direct sums in (3) only depend on the class $\bar{\chi}$ of $\chi$ in $k / \mathbb{Z}$. If $\bar{\chi}=0+$ $\mathbb{Z}$ we will assume that $\chi=0$. Let $\mathfrak{B}_{i} \subset \mathfrak{B}^{\prime}$ denote the $k[s]$-submodule generated by $\frac{1}{s-i}$. Then $\mathfrak{B}^{\prime} / \mathfrak{B}_{i}$ is isomorphic to the direct sum of skyscraper modules $k[s] /(s-j)$ for integers $j \neq i$. Thus $\left(\mathfrak{B}^{\prime} / \mathfrak{B}_{i}\right) \otimes_{k[s]} k[s] /(s-\chi-i)^{n}=0$. On the other hand $\mathfrak{B}_{i}$ is free, so $\mathfrak{B}^{\prime} \otimes_{k[s]} k[s] /(s-\chi-i)^{n}$ is free with generator $\frac{1}{s-i} \otimes 1$. These basis elements give our desired isomorphism, which evidently commutes with the action of $T$.

\subsection{Monodromization}

The $\mathbb{G}_{m}$-action on $V$ induces an algebra map $k[s] \rightarrow \mathcal{D}_{V}$, where $s=x \partial_{x}$ is the invariant vector field on $\mathbb{G}_{m}$. We say that $M \in \mathcal{M}(V)$ is monodromic if every local section $m \in M$ is killed by some nonzero polynomial in $s=$ $x \partial_{x}$. In other words, $M$ is monodromic if it is a torsion module over $k[s]$. This definition of monodromic was introduced by Verdier [14]. Define an object of $\operatorname{D\mathcal {M}}(V)$ to be monodromic if each of its cohomology $\mathcal{D}$-modules is monodromic. We denote this full subcategory by $D_{\text {mon }} \mathcal{M}(V) \subset D \mathcal{M}(V)$. 
For any $\chi \in k$ and $n \in \mathbb{N}$, let $A_{\chi, n} \subset k(s)$ consist of those rational functions with poles of order $\leq n$ at $\chi+\mathbb{Z}$ and no other poles. Define $I_{\chi}^{0, n} \in$ $\mathcal{M}\left(\mathbb{G}_{m}\right)$ to be the inverse Mellin transform $\mathfrak{M}^{-1}\left(A_{\chi, n} / k[s]\right)$. The inclusions $A_{\chi, n} \rightarrow A_{\chi, n+1}$ induce morphisms $I_{\chi}^{0, n} \rightarrow I_{\chi}^{0, n+1}$, which form an inductive system of $\mathcal{D}$-modules. Define

$$
I^{0}=\bigoplus_{\bar{\chi} \in k / \mathbb{Z}} \underset{n}{\lim } I_{\chi}^{0, n} \in \mathcal{M}\left(\mathbb{G}_{m}\right)
$$

where $\chi \in k$ is any lift of $\bar{\chi}$. It follows that $\mathfrak{M}\left(I^{0}\right)=k(s) / k[s]$.

Let $\underline{1}$ be the unit object in the monoidal category $\operatorname{D\mathcal {M}}\left(\mathbb{G}_{m}\right)$, so $\mathfrak{M}(\underline{1})=$ $k[s]$. The canonical extension of $k(s) / k[s]$ by $k[s]$ defines an extension of $I^{0}$ by $\underline{1}$ and therefore a morphism

$$
\varepsilon: I^{0} \rightarrow \underline{1}[1]
$$

The monoidal category $\operatorname{D\mathcal {M}}\left(\mathbb{G}_{m}\right)$ acts on $D \mathcal{M}(V)$ by convolution (without compact support).

Lemma 5.6. An object $M \in D \mathcal{M}(V)$ is monodromic if and only if the morphism $I^{0} * M \rightarrow M[1]$ induced by $\varepsilon$ is an isomorphism.

Proof. A calculation using the relative de Rham complex with respect to the action map $\mathbb{G}_{m} \times V \rightarrow V$ shows that for any $M \in D \mathcal{M}(V)$ and $N \in$ $\operatorname{D\mathcal {M}}\left(\mathbb{G}_{m}\right)$, there is a canonical isomorphism $N * M \cong \mathfrak{M}(N) \otimes_{k[s]} M$ in the derived category of (sheaves of) $k[s]$-modules. This implies that the cocone of the morphism $I^{0} * M \rightarrow M[1]$ is isomorphic (in the derived category of $k[s]$-modules) to $k(s) \otimes_{k[s]} M$. But $k(s)$ is flat over $k[s]$, so the vanishing of the cohomologies of $k(s) \otimes_{k[s]} M$ is equivalent to the cohomologies of $M$ being torsion modules over $k[s]$.

See [3], [11], and [7, C.2] for further details in the unipotently monodromic case (when $\chi=1$ ).

Lemma 5.7. There exists an inductive system of isomorphisms

$$
I_{\chi}^{0, n} * B \cong j_{!} I_{\chi}^{0, n} \cong I_{\chi}^{0, n} * \mathcal{L}
$$

Proof. Since $h_{*} B=h_{*} \mathcal{L}=0$, it suffices as in Lemma 3.9 to give isomorphisms of the above objects after restriction to $\mathbb{G}_{m}$. In fact, it suffices to construct isomorphisms between the Mellin transforms of these restrictions, 
i.e., isomorphisms $\mathfrak{M}\left(I_{\chi}^{0, n} * j^{*} B\right) \cong \mathfrak{M}\left(I_{\chi}^{0, n}\right) \cong \mathfrak{M}\left(I_{\chi}^{0, n} * j^{*} \mathcal{L}\right)$. This is equivalent to constructing isomorphisms

$$
\begin{array}{ll}
\mathfrak{M}\left(I_{\chi}^{0, n}\right) \underset{k[s]}{\otimes} \mathfrak{B} \cong \mathfrak{M}\left(I_{\chi}^{0, n}\right), \quad \mathfrak{B}:=\mathfrak{M}\left(j^{*} B\right), \\
\mathfrak{M}\left(I_{\chi}^{0, n}\right) \underset{k[s]}{\otimes} E \cong \mathfrak{M}\left(I_{\chi}^{0, n}\right), \quad E:=\mathfrak{M}\left(j^{*} \mathcal{L}\right)
\end{array}
$$

Note that we have isomorphisms

$$
\mathfrak{M}\left(I_{\chi}^{0, n}\right)=A_{\chi, n} / k[s] \cong \bigoplus_{i \in \mathbb{Z}} k[s] /(s-\chi-i)^{n} .
$$

Combining (5.7.3) and Proposition 5.3(3), one gets (5.7.1). Let us construct (5.7.2). We have

$$
E=\mathcal{D} /\left(1-T^{-1} s\right) \mathcal{D}
$$

Let 1 be the generator of $E$. Let $E_{i} \subset E$ denote the free $k[s]$-submodule generated by $1 T^{-i-1}$ for $i \in \mathbb{Z}$. If $\chi \in \mathbb{Z}$, set $\chi=0$. From the relation $1 T^{-i}=$ $1 T^{-i-1}(s-i)$, we deduce that $E / E_{i}$ is supported away from $\chi+i$, so $\left(E / E_{i}\right) \otimes_{k[s]} k[s] /(s-\chi-i)^{n}=0$. Hence $E \otimes_{k[s]} k[s] /(s-\chi-i)^{n}$ is freely generated by $1 T^{-i-1} \otimes 1$, and this gives us (5.7.2).

Lemma 5.7 implies in particular that $I^{0} * B \cong I^{0} * \mathcal{L}$. We deduce from Lemma 5.6 that $\operatorname{Four}_{V / S, B}$ agrees with $\operatorname{Four}_{V / S, \mathcal{L}}$ on $D_{\operatorname{mon}} \mathcal{M}(V)$.

Corollary 5.8. There is an isomorphism

$$
\operatorname{Four}_{V / S, B} \cong \operatorname{Four}_{V / S, \mathcal{L}}
$$

of functors $D_{\text {mon }} \mathcal{M}(V) \rightarrow D_{\text {mon }} \mathcal{M}\left(V^{\vee}\right)$.

\section{Appendix A. The monodromic subcategory}

In this appendix we prove the facts we need about (non-unipotently) monodromic complexes. For a more complete account of the unipotently monodromic story, see $[3,5]$.

\section{A.1. Free monodromic objects}

Let $p$ be the characteristic of $k$, which may be 0 . For $p \nmid n$, let $A_{n}^{0}$ be the group algebra $R\left[\mu_{n}\right]$ considered as a sheaf on $\operatorname{Spec} k$, i.e., a $\operatorname{Gal}(\bar{k} / k)$-module. 
Put

$$
A^{0}={\underset{\varliminf}{p \nmid n}}_{\lim _{n}} A_{n}^{0} .
$$

Consider $\mathbb{T}:=\lim _{\longleftarrow} \mu_{n}(\bar{k})$ the tame fundamental group of $\mathbb{G}_{m, \bar{k}}$. For any $\gamma \in \mathbb{T}$, let $\widetilde{\gamma}$ denote the corresponding invertible element in $A^{0}(\bar{k})$. Pick a topological generator $t \in \mathbb{T}$. Note that $\tilde{t}-1$ is not a zero divisor in $A^{0}$, so $A^{0}$ injects to the localization $A=\left(A^{0}\right)_{\tilde{t}-1}$. Define

$$
A^{i}=(\widetilde{t}-1)^{i} A^{0} \subset A
$$

for $i \in \mathbb{Z}$ and set $A_{n}^{i}=A^{i} \otimes_{A^{0}} A_{n}^{0}$ for $p \nmid n$. The definition of $A^{i}$ is independent of the choice of $t$, and $A^{i}$ is a $\operatorname{Gal}(\bar{k} / k)$-module. Note that $A^{1}$ is the kernel of the quotient map $A^{0} \rightarrow A_{1}^{0}=R$.

Remark A.2. The ring $A^{0}(\bar{k})$ is isomorphic to the product of the completions of $R\left[t, t^{-1}\right]$ at all maximal ideals $\mathfrak{m}$ such that $t^{n} \equiv 1 \bmod \mathfrak{m}$ for some $p \nmid n$. The maximal ideals $\mathfrak{m}$ correspond to the eigenvalues of the monodromy action.

For $i \in \mathbb{Z}$ and $p \nmid n$, let $I_{n}^{i}$ be the local system on $\mathbb{G}_{m}$ such that the fiber at $1 \in \mathbb{G}_{m}(k)$ is $A_{n}^{i}$ and the monodromy action of $\gamma \in \mathbb{T}$ is multiplication by $\widetilde{\gamma}$. We define $I^{i}$ to be the pro-sheaf

$$
\text { "limp" } I_{p \nmid n}^{i}
$$

where we use "lim" to denote pro-objects, following the notation of $[1$, Exposé I, (8.5.3.2)].

Remark A.3. After base change from Spec $k$ to Spec $\bar{k}$, the local systems $I_{n}^{0}$ and $I_{n}^{i}$ are isomorphic via multiplication by $(\widetilde{t}-1)^{i}$, and this induces an isomorphism $I^{0} \cong I^{i}$. The isomorphism is not canonical as it depends on the choice of $t$.

Lemma A.4. There is a canonical isomorphism of pro-objects

$$
I^{0} * M \cong M(-1)[-2]
$$

for $M \in D_{\text {mon }}^{b}(V)$ considered as a constant pro-object. 
Proof. Let $e_{n}: \mathbb{G}_{m} \rightarrow \mathbb{G}_{m}$ denote the $n^{\text {th }}$ power map. Note that $e_{n !} R \cong I_{n}^{0}$ for $p \nmid n$. Since $M$ is monodromic, there exists $n_{0}$ coprime to $p$ such that $\theta\left(n_{0}\right)^{*} M \cong \operatorname{pr}_{2}^{*} M$. Then

$$
\text { "lim" }\left(e_{n !} R\right) * M \cong \text { "lim" } \theta(n) ! \operatorname{pr}_{2}^{*} M \cong M(-1)[-2],
$$

where we use the fact that the pro-object "lim" $\Gamma_{c}\left(\mathbb{G}_{m}, R\right)$ is essentially constant and isomorphic to $R(-1)[-2]$ (cf. [13, Lemme 5.2]).

\section{A.5. Monodromic sheaves as $A^{0}$-modules}

Let $\operatorname{Mod}_{\tau}\left(A^{0}\right)$ denote the abelian category of sheaves of discrete $A^{0}$-modules on Spec $k$, where $A^{0}$ is equipped with the projective limit topology, and let $\operatorname{Sh}\left(\mathbb{G}_{m}\right)$ denote the abelian category of sheaves of $R$-modules on $\mathbb{G}_{m}$. We have a canonical exact functor

$$
\operatorname{Loc}: \operatorname{Mod}_{\tau}\left(A^{0}\right) \rightarrow \operatorname{Sh}\left(\mathbb{G}_{m}\right)
$$

Define another functor $\mathfrak{M}: \operatorname{Sh}\left(\mathbb{G}_{m}\right) \rightarrow \operatorname{Mod}_{\tau}\left(A^{0}\right)$ by

$$
\mathfrak{M}(\mathcal{F})=\lim _{\longrightarrow} h_{*}^{\prime} e_{n, *} e_{n}^{*} \mathcal{F}
$$

where $h^{\prime}: \mathbb{G}_{m} \rightarrow$ Spec $k$ is the structure map and $A^{0}$ acts on $e_{n, *} e_{n}^{*} \mathcal{F}$ by transport of structure. We deduce from étale descent that Loc is left adjoint to $\mathfrak{M}$. Passing to derived categories, the derived functors are still adjoint, and we also denote them by

$$
\operatorname{Loc}: D \operatorname{Mod}_{\tau}\left(A^{0}\right) \leftrightarrows D\left(\mathbb{G}_{m}\right): \mathfrak{M}
$$

Note that $\mathfrak{M}: D\left(\mathbb{G}_{m}\right) \rightarrow D \operatorname{Mod}_{\tau}\left(A^{0}\right)$ is equal to the composition of the exact functor $\lim _{\longrightarrow} e_{n, *} e_{n}^{*}$ with the derived functor $h_{*}^{\prime}$.

Proposition A.6. The derived functor $\operatorname{Loc}: D \operatorname{Mod}_{\tau}\left(A^{0}\right) \rightarrow D\left(\mathbb{G}_{m}\right)$ is fully faithful.

Proof. We need to show that the unit of adjunction $L \rightarrow \mathfrak{M} \circ \operatorname{Loc}(L)$ is an isomorphism for $L \in \operatorname{DMod}_{\tau}\left(A^{0}\right)$. We can assume that $k$ is algebraically closed. Since Loc and $\mathfrak{M}$ both commute with filtered colimits, we may further suppose that $L$ is a finite module concentrated in degree 0 . Then there exists $n_{0}$ not divisible by $p$ such that the action of $A^{0}$ on $L$ factors through $R\left[\mu_{n_{0}}\right]$. If $n$ is a multiple of $n_{0}$ then $e_{n}^{*} \operatorname{Loc}(L) \cong \underline{L}$, where $\underline{L}$ is the constant sheaf 
on $\mathbb{G}_{m}$ with stalk $L$. The proposition now follows from the fact that for any finite abelian group $L$ of order prime to $p$, one has $\underline{\lim } H^{0} \Gamma\left(\mathbb{G}_{m}, e_{n, *} e_{n}^{*} \underline{L}\right) \cong \underline{L}$ and $\lim _{\longrightarrow} H^{i} \Gamma\left(\mathbb{G}_{m}, e_{n, *} e_{n}^{*} \underline{L}\right)=0$ for $i \neq 0$.

Corollary A.7. The restriction of Loc induces an equivalence between the subcategory of $D^{b} \operatorname{Mod}_{\tau}\left(A^{0}\right)$ consisting of complexes whose cohomology sheaves have finite stalks and $D_{\text {mon }}^{b}\left(\mathbb{G}_{m}\right)$. Taking hearts with respect to the standard t-structures of the above triangulated categories, we get an isomorphism between the abelian category of sheaves of $A^{0}$-modules on Spec $k$ with finite stalk and the abelian category of monodromic sheaves on $\mathbb{G}_{m}$.

The monoidal structure on $D \operatorname{Mod}_{\tau}\left(A^{0}\right)$ with respect to (derived) tensor product over $A^{0}$ corresponds under Loc to convolution on $D\left(\mathbb{G}_{m}\right)$.

Lemma A.8. For $L, K \in D \operatorname{Mod}_{\tau}\left(A^{0}\right)$ there exists a canonical isomorphism

$$
\operatorname{Loc}(L) * \operatorname{Loc}(K) \cong \operatorname{Loc}\left(L \underset{A^{0}}{\otimes} K\right)(-1)[-2] .
$$

Proof. Consider the functor $\operatorname{Loc}_{\mathbb{G}_{m} \times \mathbb{G}_{m}}: D \operatorname{Mod}_{\tau}\left(A^{0} \widehat{\otimes}_{R} A^{0}\right) \rightarrow D\left(\mathbb{G}_{m} \times \mathbb{G}_{m}\right)$, which is defined similarly to the above functor $\operatorname{Loc}=\operatorname{Loc}_{\mathbb{G}_{m}}$. Applying $\operatorname{Loc}_{\mathbb{G}_{m}} \times \mathbb{G}_{m}$ to the natural map $L \otimes_{R} K \rightarrow L \otimes_{A^{0}} K$, we get a map

$$
\operatorname{Loc}(L) \otimes \operatorname{Loc}(K) \rightarrow m^{*} \operatorname{Loc}\left(L \underset{A^{0}}{\otimes} K\right)
$$

in $D\left(\mathbb{G}_{m} \times \mathbb{G}_{m}\right)$. Recall that since $m$ is smooth, $m^{*} \operatorname{Loc}\left(L \otimes_{A^{0}} K\right)$ is isomorphic to $m^{!} \operatorname{Loc}\left(L \otimes_{A^{0}} K\right)(-1)[-2]$. Therefore the $\left(m_{!}, m^{!}\right)$-adjunction induces a morphism

$$
\operatorname{Loc}(L) * \operatorname{Loc}(K) \rightarrow \operatorname{Loc}\left(L \underset{A^{0}}{\otimes} K\right)(-1)[-2] .
$$

To check this is an isomorphism, we can assume $k$ is algebraically closed and take $L=K=A_{n}^{0}$ for $p \nmid n$ since the functors on both sides commute with filtered colimits and have finite cohomological amplitude. Under these assumptions, the isomorphism is an easy computation.

Corollary A.9. There is a canonical projective system of isomorphisms

$$
I^{i} * I_{n}^{j} \cong I_{n}^{i+j}(-1)[-2]
$$

for $p \nmid n$ and any integers $i$ and $j$. Consequently there is an isomorphism of pro-objects

$$
I^{i} * I^{j} \cong I^{i+j}(-1)[-2]
$$


Proof. Fix $p \nmid n$. By Lemma A.8, the first isomorphism is equivalent to an isomorphism

$$
\underbrace{}_{p \nmid m} \lim " A_{m}^{i}{\underset{A}{A^{0}}}_{A_{n}} A_{n}^{j} A_{n}^{i+j}
$$

as pro-objects in $D \operatorname{Mod}_{\tau}\left(A^{0}\right)$. Remark A.3 and Lemma A.4 imply that it suffices to consider the cohomology in degree 0 , i.e., we consider the nonderived tensor product on the LHS. Then $H^{0}\left(A_{m}^{i} \otimes_{A^{0}} A_{n}^{j}\right) \cong A_{n}^{i+j}$ for $n \mid m$ by definition. These isomorphisms are evidently compatible with changes in $n$, so the rest of the corollary follows.

\section{Acknowledgements}

The research was partially supported by the Department of Defense (DoD) through the NDSEG fellowship. The author is very thankful to Sasha Beilinson and Vladimir Drinfeld for many helpful discussions. The definition of $\operatorname{Four}_{B}$ was first suggested by Drinfeld.

\section{References}

[1] Théorie des topos et cohomologie étale des schémas. Tome 1: Théorie des topos, Lecture Notes in Mathematics, Vol. 269, Springer-Verlag, Berlin-New York (1972). Séminaire de Géométrie Algébrique du BoisMarie 1963-1964 (SGA 4), Dirigé par M. Artin, A. Grothendieck, et J. L. Verdier. Avec la collaboration de N. Bourbaki, P. Deligne et B. Saint-Donat.

[2] Cohomologie l-adique et fonctions L, Lecture Notes in Mathematics, Vol. 589, Springer-Verlag, Berlin (1977), ISBN 3-540-08248-4. Séminaire de Géometrie Algébrique du Bois-Marie 1965-1966 (SGA 5), Edité par Luc Illusie.

[3] A. Beilinson, How to glue perverse sheaves. In: K-theory, arithmetic and geometry (Moscow, 1984-1986), Vol. 1289 of Lecture Notes in Math., 42-51, Springer, Berlin (1987).

[4] A. Beilinson, A remark on primitive cycles and Fourier-Radon transform. In: Regulators, Vol. 571 of Contemp. Math., 19-23, Amer. Math. Soc., Providence, RI (2012).

[5] R. Bezrukavnikov and Z. Yun, On Koszul duality for Kac-Moody groups. 
[6] P. Deligne, Cohomologie étale, Lecture Notes in Mathematics, Vol. 569, Springer-Verlag, Berlin (1977). Séminaire de Géométrie Algébrique du Bois-Marie SGA $4 \frac{1}{2}$, Avec la collaboration de J. F. Boutot, A. Grothendieck, L. Illusie et J. L. Verdier.

[7] V. Drinfeld and D. Gaitsgory, Compact generation of the category of $D$-modules on the stack of G-bundles on a curve. arXiv:1112.2402v6.

[8] N. M. Katz and G. Laumon, Transformation de Fourier et majoration de sommes exponentielles. Inst. Hautes Études Sci. Publ. Math., (1985), no. $62,361-418$.

[9] R. Kiehl and R. Weissauer, Weil conjectures, perverse sheaves and l'adic Fourier transform. Vol. 42 of Ergebnisse der Mathematik und ihrer Grenzgebiete. 3. Folge. A Series of Modern Surveys in Mathematics [Results in Mathematics and Related Areas. 3rd Series. A Series of Modern Surveys in Mathematics], Springer-Verlag, Berlin (2001), ISBN 3-540-41457-6.

[10] G. Laumon, Transformation de Fourier homogène. Bull. Soc. Math. France, 131 (2003), no. 4, 527-551.

[11] S. Lichtenstein, Vanishing cycles for algebraic D-modules. Harvard senior thesis, March 2009.

[12] T. A. Springer, A purity result for fixed point varieties in flag manifolds. J. Fac. Sci. Univ. Tokyo Sect. IA Math., 31 (1984), no. 2, 271-282.

[13] J.-L. Verdier, Spécialisation de faisceaux et monodromie modérée. In: Analysis and topology on singular spaces, II, III (Luminy, 1981), Vol. 101 of Astérisque, 332-364, Soc. Math. France, Paris (1983).

[14] J.-L. Verdier, Prolongement des faisceaux pervers monodromiques. Astérisque (1985), no. 130, 218-236. Differential systems and singularities (Luminy, 1983).

Department of Mathematics, University of Chicago

5734 S. University Avenue, Room 208C, Chicago, Illinois 60637, USA

E-mail address: jpwang@math.uchicago.edu

RECEIVED April 17, 2014 